\title{
Resolvent for Non-Self-Adjoint Differential Operator with Block-Triangular Operator Potential
}

\author{
Aleksandr Mikhailovich Kholkin \\ Department of Higher and Applied Mathematics, Priazovskyi State Technical University, Universitetskaya Street 7 , \\ Mariupol 87500, Ukraine
}

Correspondence should be addressed to Aleksandr Mikhailovich Kholkin; a.kholkin@gmail.com

Received 10 July 2016; Revised 30 September 2016; Accepted 6 October 2016

Academic Editor: Cemil Tunç

Copyright (C) 2016 Aleksandr Mikhailovich Kholkin. This is an open access article distributed under the Creative Commons Attribution License, which permits unrestricted use, distribution, and reproduction in any medium, provided the original work is properly cited.

A resolvent for a non-self-adjoint differential operator with a block-triangular operator potential, increasing at infinity, is constructed. Sufficient conditions under which the spectrum is real and discrete are obtained.

\section{Introduction}

The theory of non-self-adjoint singular differential operators, generated by scalar differential expressions, has been well studied. An overview on the theory of non-self-adjoint singular ordinary differential operators is provided in V. E. Lyantse's Appendix I to the monograph of Naimark [1]. In this regard the papers of Naimark [2], Lyantse [3], Marchenko [4], Rofe-Beketov [5], Schwartz [6], and Kato [7] should be noted. The questions regarding equations with nonHermitian matrix or operator coefficients have been studied insufficiently. For a differential operator with a triangular matrix potential decreasing at infinity, which has a bounded first moment due to the inverse scattering problem, it is stated in $[8,9]$ that the discrete spectrum of the operator consists of a finite number of negative eigenvalues, and the essential spectrum covers the positive semiaxis. The questions regarding an operator with a block-triangular matrix potential that increases at infinity are considered in $[10,11]$. In the future, by the author of this paper similar questions are considered for equations with block-triangular operator coefficients. In $[11,12]$ Green's function of a non-self-adjoint operator is constructed.

In this article we construct a resolvent for a non-selfadjoint differential operator, using which the structure of the operator spectrum is set.

\section{Preliminary Notes}

Let $H_{k}, k=1,2, \ldots, r$, be finite-dimensional or infinitedimensional separable Hilbert space with inner product $(\cdot, \cdot)$ and norm $|\cdot|, \operatorname{dim} H_{k} \leq \infty$. Denote $\mathbf{H}=H_{1} \oplus H_{2} \oplus$ $\cdots \oplus H_{r}$. Element $h \in \mathbf{H}$ will be written in the form $h=$ $\operatorname{col}\left(h_{1}, h_{2}, \ldots, h_{r}\right)$, where $h_{k} \in H_{k}, k=\overline{1, r}, I_{k}, I$ are identity operators in $H_{k}$ and $\mathbf{H}$ accordingly.

We denote by $L_{2}(\mathbf{H},(0, \infty))$ the Hilbert space of vectorvalued functions $y(x)$ with values in $\mathbf{H}$ with inner product

$$
\langle y, z\rangle=\int_{0}^{\infty}(y(x), z(x)) d x
$$

and the corresponding norm $\|\cdot\|$.

Consider the equation with block-triangular operator potential

$$
l[y]=-y^{\prime \prime}+V(x) y=\lambda y, \quad 0 \leq x<\infty,
$$

where

$$
\begin{aligned}
& V(x)=v(x) \cdot I+U(x) \\
& U(x)=\left(\begin{array}{cccc}
U_{11}(x) & U_{12}(x) & \cdots & U_{1 r}(x) \\
0 & U_{22}(x) & \cdots & U_{2 r}(x) \\
\cdots & \cdots & \cdots & \cdots \\
0 & 0 & \cdots & U_{r r}(x)
\end{array}\right),
\end{aligned}
$$


$v(x)$ is a real scalar function, and $0<v(x) \rightarrow \infty$ monotonically, as $x \rightarrow \infty$, and it has monotone absolutely continuous derivative. Also, $U(x)$ is a relatively small perturbation; for example, $|U(x)| \cdot v^{-1}(x) \rightarrow 0$ as $x \rightarrow \infty$ or $|U| v^{-1} \in L^{\infty}\left(\mathbb{R}_{+}\right)$. The diagonal blocks $U_{k k}(x), k=\overline{1, r}$, are assumed as bounded self-adjoint operators in $H_{k}, U_{k l}: H_{l} \rightarrow H_{k}$.

In case where

$$
v(x) \geq C x^{2 \alpha}, \quad C>0, \alpha>1,
$$

we suppose that coefficients of (2) satisfy relations

$$
\begin{aligned}
& \int_{0}^{\infty}|U(t)| \cdot v^{-1 / 2}(t) d t<\infty \\
& \int_{0}^{\infty} v^{\prime 2}(t) \cdot v^{-5 / 2}(t) d t<\infty \\
& \int_{0}^{\infty} v^{\prime \prime}(t) \cdot v^{-3 / 2}(t) d t<\infty
\end{aligned}
$$

Let us consider the functions

$$
\begin{gathered}
\gamma_{0}(x)=\frac{1}{\sqrt[4]{4 v(x)}} \cdot \exp \left(-\int_{0}^{x} \sqrt{v(u)} d u\right), \\
\gamma_{\infty}(x)=\frac{1}{\sqrt[4]{4 v(x)}} \cdot \exp \left(\int_{0}^{x} \sqrt{v(u)} d u\right) .
\end{gathered}
$$

It is easy to see that $\gamma_{0}(x) \rightarrow 0, \gamma_{\infty}(x) \rightarrow \infty$ as $x \rightarrow \infty$. These solutions constitute a fundamental system of solutions of the scalar differential equation

$$
-z^{\prime \prime}+(v(x)+q(x)) z=0,
$$

where $q(x)$ is determined by a formula (cf. with the monograph [13])

$$
q(x)=\frac{5}{16}\left(\frac{v^{\prime}(x)}{v(x)}\right)^{2}-\frac{1}{4} \frac{v^{\prime \prime}(x)}{v(x)} .
$$

In such a way for all $x \in[0, \infty)$ one has

$$
W\left(\gamma_{0}, \gamma_{\infty}\right):=\gamma_{0}(x) \cdot \gamma_{\infty}^{\prime}(x)-\gamma_{0}^{\prime}(x) \cdot \gamma_{\infty}(x)=1 .
$$

In case of $v(x)=x^{2 \alpha}, 0<\alpha \leq 1$, we suppose that the coefficients of (2) satisfy the relation

$$
\int_{a}^{\infty}|U(t)| \cdot t^{-\alpha} d t<\infty, \quad a>0 .
$$

Now functions $\gamma_{0}(x, \lambda)$ and $\gamma_{\infty}(x, \lambda)$ are defined as follows:

$$
\begin{aligned}
& \gamma_{0}(x, \lambda)=\frac{1}{\sqrt[4]{4\left(x^{2 \alpha}-\lambda\right)}} \cdot \exp \left(-\int_{a}^{x} \sqrt{u^{2 \alpha}-\lambda} d u\right), \\
& \gamma_{\infty}(x, \lambda)=\frac{1}{\sqrt[4]{4\left(x^{2 \alpha}-\lambda\right)}} \cdot \exp \left(\int_{a}^{x} \sqrt{u^{2 \alpha}-\lambda} d u\right) .
\end{aligned}
$$

These functions also form a fundamental system of solutions of the scalar differential equation, which is obtained by replacing $v(x)$ with $v(x)-\lambda$ in formulas (7) and (8).
In [10] the asymptotic behavior of the functions $\gamma_{0}(x, \lambda)$ and $\gamma_{\infty}(x, \lambda)$ was established as $x \rightarrow \infty$. If $(\alpha+1) / 2 \alpha=n \in \mathbb{N}$, that is, $\alpha=1 /(2 n-1)$, then functions $\gamma_{0}(x, \lambda)$ and $\gamma_{\infty}(x, \lambda)$ as $x \rightarrow \infty$ will have the following asymptotic behavior:

$$
\begin{aligned}
& \gamma_{0}(x, \lambda)=c \cdot \exp \left(-\frac{x^{1+\alpha}}{1+\alpha}+\frac{\lambda}{2} \cdot \frac{x^{1-\alpha}}{1-\alpha}\right. \\
& \left.+\sum_{k=2}^{n-1} \frac{1 \cdot 3 \cdot \ldots \cdot(2 k-3)}{k !} \cdot\left(\frac{\lambda}{2}\right)^{k} \cdot \frac{x^{1-(2 k-1) \alpha}}{1-(2 k-1) \alpha}\right) \\
& \cdot x^{((1 \cdot 3 \cdot \ldots \cdot(2 n-3)) / n !) \cdot(\lambda / 2)^{n}-\alpha / 2} \cdot(1+o(1)), \\
& \gamma_{\infty}(x, \lambda)=c \cdot \exp \left(\frac{x^{1+\alpha}}{1+\alpha}-\frac{\lambda}{2} \cdot \frac{x^{1-\alpha}}{1-\alpha}\right. \\
& \left.-\sum_{k=2}^{n-1} \frac{1 \cdot 3 \cdot \ldots \cdot(2 k-3)}{k !} \cdot\left(\frac{\lambda}{2}\right)^{k} \cdot \frac{x^{1-(2 k-1) \alpha}}{1-(2 k-1) \alpha}\right) \\
& \cdot x^{-\left(((1 \cdot 3 \cdot \ldots \cdot(2 n-3)) / n !) \cdot(\lambda / 2)^{n}+\alpha / 2\right)} \cdot(1+o(1)) .
\end{aligned}
$$

In particular, with $\alpha=1(n=1)$ one has

$$
\begin{aligned}
& \gamma_{0}(x, \lambda)=c \cdot x^{(\lambda-1) / 2} \cdot \exp \left(-\frac{x^{2}}{2}\right)(1+o(1)), \\
& \gamma_{\infty}(x, \lambda)=c \cdot x^{-(\lambda+1) / 2} \cdot \exp \left(\frac{x^{2}}{2}\right)(1+o(1)) .
\end{aligned}
$$

In the case $(\alpha+1) / 2 \alpha \notin \mathbb{N}$, set $n=[(\alpha+1) / 2 \alpha]+1$, with $[\beta]$ being the integral part of $\beta$, to obtain the following asymptotic behavior for $\gamma_{0}(x, \lambda)$ and $\gamma_{\infty}(x)$ at infinity:

$$
\begin{aligned}
& \gamma_{0}(x, \lambda)=c \cdot x^{-\alpha / 2} \exp \left(-\frac{x^{1+\alpha}}{1+\alpha}+\frac{\lambda}{2} \cdot \frac{x^{1-\alpha}}{1-\alpha}\right. \\
&\left.+\sum_{k=2}^{n-1} \frac{1 \cdot 3 \cdot \ldots \cdot(2 k-3)}{k !} \cdot\left(\frac{\lambda}{2}\right)^{k} \cdot \frac{x^{1-(2 k-1) \alpha}}{1-(2 k-1) \alpha}\right) \\
& \cdot \exp \left(-\frac{1 \cdot 3 \cdot \ldots \cdot(2 n-3)}{n !} \cdot\left(\frac{\lambda}{2}\right)^{n} \cdot \frac{x^{-\alpha}}{\alpha}\right) \cdot(1 \\
&\left.+o\left(x^{-\alpha}\right)\right), \quad \frac{x^{1+\alpha}}{1+\alpha}-\frac{\lambda}{2} \cdot \frac{x^{1-\alpha}}{1-\alpha} \\
&\left.\gamma_{\infty}(x, \lambda)=c \cdot x^{-\alpha / 2} \exp \left(\frac{\lambda}{2}\right)^{k} \cdot \frac{x^{1-(2 k-1) \alpha}}{1-(2 k-1) \alpha}\right) \\
&\left.\quad-\sum_{k=2}^{n-1} \frac{1 \cdot 3 \cdot \ldots \cdot(2 k-3)}{k !} \cdot\left(\frac{\lambda}{2}\right)^{n} \cdot \frac{x^{-\alpha}}{\alpha}\right) \cdot(1 \\
&+ \exp \left(\frac{1 \cdot 3 \cdot \ldots \cdot(2 n-3)}{n !}+o\left(x^{-\alpha}\right)\right) \cdot\left(\frac{1}{\alpha}\right)
\end{aligned}
$$

In [10] for an equation with matrix coefficients, and in the furtherance for equations with operator coefficients, the following theorem is proved. 
Theorem 1. If for (2) conditions (4)-(5) are satisfied for $\alpha>1$ or condition (10) for $0<\alpha \leq 1$, then the equation has a unique decreasing at infinity operator solution $\Phi(x, \lambda)$, satisfying the conditions

$$
\begin{aligned}
& \lim _{x \rightarrow \infty} \frac{\Phi(x, \lambda)}{\gamma_{0}(x, \lambda)}=I, \\
& \lim _{x \rightarrow \infty} \frac{\Phi^{\prime}(x, \lambda)}{\gamma_{0}^{\prime}(x, \lambda)}=I .
\end{aligned}
$$

Also, there exists increasing at infinity operator solution $\Psi(x, \lambda)$, satisfying the conditions

$$
\begin{aligned}
& \lim _{x \rightarrow \infty} \frac{\Psi(x, \lambda)}{\gamma_{\infty}(x, \lambda)}=I, \\
& \lim _{x \rightarrow \infty} \frac{\Psi^{\prime}(x, \lambda)}{\gamma_{\infty}^{\prime}(x, \lambda)}=I .
\end{aligned}
$$

Corollary 2. If $\alpha=1$, that is, $v(x)=x^{2}$, then, under condition (10), the solutions $\Phi(x, \lambda)$ and $\Psi(x, \lambda)$ have common (known) asymptotic behavior, as in the quality $\gamma_{0}(x, \lambda)$ and $\gamma_{\infty}(x, \lambda)$ you can take the following functions:

$$
\begin{aligned}
& \gamma_{0}(x, \lambda)=x^{(\lambda-1) / 2} \cdot \exp \left(-\frac{x^{2}}{2}\right), \\
& \gamma_{\infty}(x, \lambda)=x^{-(\lambda+1) / 2} \cdot \exp \left(\frac{x^{2}}{2}\right) .
\end{aligned}
$$

\section{Resolvent of the Non-Self-Adjoint Operator}

Let the following boundary condition be given at $x=0$ :

$$
\cos A \cdot y^{\prime}(0)-\sin A \cdot y(0)=0
$$

where $A$ is block-triangular operator of the same structure as the potential $V(x)$ (3) of the differential equation (2), and $A_{k k}, k=\overline{1, r}$, are the bounded self-adjoint operators in $H_{k}$, which satisfy the conditions

$$
-\frac{\pi}{2} I_{k} \ll A_{k k} \leq \frac{\pi}{2} I_{k}
$$

Together with problem (2) and (18) we consider the separated system

$$
\begin{aligned}
& l_{k}\left[y_{k}\right]=-y_{k}^{\prime \prime}+\left(v(x) I_{k}+U_{k k}(x)\right) y_{k}=\lambda y_{k}, \\
& k=\overline{1, r}
\end{aligned}
$$

with the boundary conditions

$$
\cos A_{k k} \cdot y_{k}^{\prime}(0)-\sin A_{k k} \cdot y_{k}(0)=0, \quad k=\overline{1, r} .
$$

Let $L^{\prime}$ denote the minimal differential operator generated by differential expression $l[y]$ and the boundary condition (18), and let $L_{k}^{\prime}, k=\overline{1, r}$, denote the minimal differential operator on $L_{2}(\mathbf{H},(0, \infty))$ generated by differential expression $l_{k}\left[y_{k}\right]$ and the boundary conditions (21). Taking into account the conditions on coefficients, as well as sufficient smallness of perturbations $U_{k k}(x)$, and conditions (19), we conclude that, for every symmetric operator $L_{k}^{\prime}, k=\overline{1, r}$, there is a case of limit point at infinity. Hence their self-adjoint extensions $L_{k}$ are the closures of operators $L_{k}^{\prime}$, respectively. The operators $L_{k}$ are semibounded below, and their spectra are discrete.

Let $L$ denote the operator extensions $L^{\prime}$, by requiring that $L_{2}(\mathbf{H},(0, \infty))$ be the domain of operator $L$.

The following theorem is proved in [10].

Theorem 3. Suppose that for (2) conditions (4)-(5) are satisfied for $\alpha>1$ or condition (10) for $0<\alpha \leq 1$. Then the discrete spectrum of the operator $L$ is real and coincides with the union of spectra of the self-adjoint operators $L_{k}, k=\overline{1, r}$; that is,

$$
\sigma_{d}(L)=\bigcup_{k=1}^{r} \sigma\left(L_{k}\right) .
$$

Comment 4. Note that this theorem contains a statement of the discrete spectrum of the non-self-adjoint operator $L$ only and no allegations of its continuous and residual spectrum.

Along with (2) we consider the equation

$$
l_{1}[y]=-y^{\prime \prime}+V^{*}(x) y=\lambda y
$$

$\left(V^{*}(x)\right.$ is adjoint to the operator $\left.V(x)\right)$. If the space $\mathbf{H}$ is finitedimensional, then (23) can be rewritten as

$$
\tilde{l}[\tilde{y}]=-\widetilde{y}^{\prime \prime}+\tilde{y} V(x)=\lambda \tilde{y},
$$

where $\tilde{y}=\left(\begin{array}{llll}\tilde{y}_{1} & \tilde{y}_{2} & \ldots & \tilde{y}_{r}\end{array}\right)$ and the equation is called the left.

For operator functions $Y(x, \lambda), Z(x, \lambda) \in B(\mathbf{H})$ let

$$
\begin{aligned}
W\left\{Z^{*}, Y\right\}= & Z^{* \prime}(x, \bar{\lambda}) Y(x, \lambda) \\
& -Z^{*}(x, \bar{\lambda}) Y^{\prime}(x, \lambda) .
\end{aligned}
$$

If $Y(x, \lambda)$ is operator solution of $(2)$ and $Z(x, \lambda)$ is operator solution of (23), the Wronskian does not depend on $x$.

Now we denote $Y(x, \lambda)$ and $Y_{1}(x, \lambda)$ as the solutions of $(2)$ and (23), respectively, satisfying the initial conditions

$$
\begin{aligned}
Y(0, \lambda) & =\cos A, \\
Y^{\prime}(0, \lambda) & =\sin A, \\
Y_{1}(0, \lambda) & =(\cos A)^{*}, \\
Y_{1}^{\prime}(0, \lambda) & =(\sin A)^{*},
\end{aligned}
$$

$$
\lambda \in \mathbb{C} .
$$

Because the operator function $Y_{1}^{*}(x, \bar{\lambda})$ satisfies equation

$$
-Y_{1}^{* \prime \prime}(x, \bar{\lambda})+Y_{1}^{*}(x, \bar{\lambda}) \cdot V(x)=\lambda Y_{1}^{*}(x, \bar{\lambda}),
$$

the operator function $\tilde{Y}(x, \lambda)=: Y_{1}^{*}(x, \bar{\lambda})$ is a solution to the left of the equation

$$
-\tilde{Y}^{\prime \prime}(x, \lambda)+\tilde{Y}(x, \lambda) \cdot V(x)=\lambda \tilde{Y}(x, \lambda)
$$

and satisfies the initial conditions $\tilde{Y}(0, \lambda)=\cos A, \tilde{Y}^{\prime}(0, \lambda)=$ $\sin A, \lambda \in \mathbb{C}$. 
Operator solutions of (23) decreasing and increasing at infinity will be denoted by $\Phi_{1}(x, \lambda), \Psi_{1}(x, \lambda)$, and the corresponding solutions of (28) are denoted by $\widetilde{\Phi}(x, \lambda)$ and $\widetilde{\Psi}(x, \lambda)$. The system operator solutions $Y(x, \lambda), \widetilde{\Phi}(x, \lambda) \in$ $B(\mathbf{H})$ of (2) and (28), respectively, will take the form of Wronskian $W\{\widetilde{\Phi}, Y\}=\widetilde{\Phi}^{\prime}(x, \lambda) Y(x, \lambda)-\widetilde{\Phi}(x, \lambda) Y^{\prime}(x, \lambda)$.

Let us designate

$$
\begin{aligned}
& G(x, t, \lambda) \\
& \quad= \begin{cases}Y(x, \lambda)(W\{\widetilde{\Phi}, Y\})^{-1} \widetilde{\Phi}(t, \lambda) & 0 \leq x \leq t \\
-\Phi(x, \lambda)(W\{\widetilde{Y}, \Phi\})^{-1} \widetilde{Y}(t, \lambda) & x \geq t .\end{cases}
\end{aligned}
$$

It is proved in [12] that the operator function $G(x, t, \lambda)$ is Green's function of the differential operator $L$; that is, it possesses all the classical properties of Green's function. In particular, for a fixed $t$ the function $G(x, t, \lambda)$ of the variable $x$ is an operator solution of (2) on each of the intervals $[0, t)$, $(t, \infty)$, and it satisfies the boundary condition (18), and at a fixed $x$, the function $G(x, t, \lambda)$ satisfies (28) in the variable $t$ on each of the intervals $[0, x),(x, \infty)$, and it satisfies the boundary condition $(\cos A)^{*} \cdot y^{\prime}(0)-(\sin A)^{*} \cdot y(0)=0$.

By definition (28), function $G(x, t, \lambda)$ is meromorphic by parameter $\lambda$ with the poles coinciding with the eigenvalues of the operator $L$.

We consider the operator $R_{\lambda}$ defined in $L_{2}(H,(0, \infty))$ by the relation

$$
\begin{aligned}
\left(R_{\lambda} f\right)(x) & =\int_{0}^{\infty} G(x, t, \lambda) f(t) d t \\
= & -\int_{0}^{x} \Phi(x, \lambda)(W\{\widetilde{Y}, \Phi\})^{-1} \widetilde{Y}(t, \lambda) f(t) d t \\
& +\int_{x}^{\infty} Y(x, \lambda)(W\{\widetilde{\Phi}, Y\})^{-1} \widetilde{\Phi}(t, \lambda) f(t) d t .
\end{aligned}
$$

Theorem 5. The operator $R_{\lambda}$ is the resolvent of the operator $L$.

\section{Proof of Theorem 5}

One can directly verify that, for any function $f(x) \epsilon$ $L_{2}(\mathbf{H},(0, \infty))$, the vector-function $y(x, \lambda)=\left(R_{\lambda} f\right)(x)$ is a solution of the equation $l[y]-\lambda y=f$ whenever $\lambda \notin \sigma(L)$. We will prove that $y(x, \lambda) \in L_{2}(\mathbf{H},(0, \infty))$.

Since operator solutions $\Phi(x, \lambda)$ and $\Psi(x, \lambda)$ form a fundamental system of solutions of $(2)$, the operator solution $Y(x, \lambda)$ of (2) satisfying the initial conditions (26) can be written as $Y(x, \lambda)=\Phi(x, \lambda) A(\lambda)+\Psi(x, \lambda) B(\lambda)$, where $A(\lambda)=$ $W\{\widetilde{\Psi}, Y\}, B(\lambda)=-W\{\widetilde{\Phi}, Y\} ;$ that is,

$$
Y(x, \lambda)=\Phi(x, \lambda) W\{\widetilde{\Psi}, Y\}-\Psi(x, \lambda) W\{\widetilde{\Phi}, Y\}
$$

Similarly, the operator solution $\tilde{Y}(x, \lambda)$ of $(28)$ can be represented in the following form:

$$
\widetilde{Y}(x, \lambda)=W\{\tilde{Y}, \Phi\} \widetilde{\Psi}(x, \lambda)-W\{\widetilde{Y}, \Psi\} \widetilde{\Phi}(x, \lambda)
$$

By using formulas (31) and (32), we can rewrite relation (30) as follows:

$$
\begin{aligned}
& \left(R_{\lambda} f\right)(x) \\
& =-\int_{0}^{a} \Phi(x, \lambda)(W\{\widetilde{Y}, \Phi\})^{-1} \tilde{Y}(t, \lambda) f(t) d t \\
& \quad+\chi_{1}(x, \lambda)-\chi_{2}(x, \lambda)+\chi_{3}(x, \lambda)-\chi_{4}(x, \lambda),
\end{aligned}
$$

where $a>0$ and

$$
\begin{gathered}
\chi_{1}(x, \lambda)=\Phi(x, \lambda)(W\{\tilde{Y}, \Phi\})^{-1} W\{\tilde{Y}, \Psi\} \\
\cdot \int_{a}^{x} \widetilde{\Phi}(t, \lambda) f(t) d t \\
\chi_{2}(x, \lambda)=\Phi(x, \lambda) \int_{a}^{x} \widetilde{\Psi}(t, \lambda) f(t) d t, \\
\chi_{3}(x, \lambda)=\Phi(x, \lambda) W\{\widetilde{\Psi}, Y\}(W\{\widetilde{\Phi}, Y\})^{-1} \\
\cdot \int_{x}^{\infty} \widetilde{\Phi}(t, \lambda) f(t) d t, \\
\chi_{4}(x, \lambda)=\Psi(x, \lambda) \int_{x}^{\infty} \widetilde{\Phi}(t, \lambda) f(t) d t .
\end{gathered}
$$

Let us show that each of these vector-functions $\chi_{1}(x, \lambda)$, $\chi_{2}(x, \lambda), \chi_{3}(x, \lambda)$, and $\chi_{4}(x, \lambda)$ belongs to $L_{2}(\mathbf{H},(0, \infty))$. Since the operator solution $\Phi(x, \lambda)$ decays fairly quickly as $x \rightarrow \infty$, then $|\Phi(x, \lambda)| \in L_{2}(0, \infty)$. It follows that

$$
\begin{aligned}
\left|\chi_{1}(x, \lambda)\right| \leq & c(\lambda) \cdot|\Phi(x, \lambda)| \cdot \int_{a}^{x}|\widetilde{\Phi}(t, \lambda)| \cdot|f(t)| d t \\
\leq & c(\lambda) \cdot|\Phi(x, \lambda)| \cdot\left(\int_{a}^{x}|\widetilde{\Phi}(t, \lambda)| d t\right)^{1 / 2} \\
& \cdot\left(\int_{a}^{x}|f(t)| d t\right)^{1 / 2} \\
< & c(\lambda) \cdot|\Phi(x, \lambda)| \cdot\left(\int_{a}^{\infty}|\widetilde{\Phi}(t, \lambda)| d t\right)^{1 / 2} \\
& \cdot\left(\int_{a}^{\infty}|f(t)| d t\right)^{1 / 2} \leq c_{1}(\lambda) \cdot|\Phi(x, \lambda)|
\end{aligned}
$$

and therefore $\chi_{1}(x, \lambda) \in L_{2}(\mathbf{H},(0, \infty))$. Similarly we get that $\chi_{3}(x, \lambda) \in L_{2}(\mathbf{H},(0, \infty))$. First we prove the assertion for the function $\chi_{2}(x, \lambda)$, when $\alpha>1$ and the coefficients of (2) satisfy the conditions (4)-(5). In this case, we have

$$
\left|\chi_{2}(x, \lambda)\right| \leq|\Phi(x, \lambda)| \int_{a}^{x}|\widetilde{\Psi}(t, \lambda)||f(t)| d t .
$$

By virtue of the asymptotic formulas for the operator solutions $\Phi(x, \lambda)$ and $\Psi(x, \lambda)$ we obtain that

$$
\left|\chi_{2}(x, \lambda)\right| \leq c_{1}(\lambda) \gamma_{0}(x, \lambda) \int_{a}^{x} \gamma_{\infty}(t, \lambda)|f(t)| d t
$$


Let us rewrite this relation in the following form:

$$
\begin{aligned}
& \left|\chi_{2}(x, \lambda)\right| \\
& \quad \leq c_{1}(\lambda) \gamma_{0}(x, \lambda) \gamma_{\infty}(x, \lambda) \int_{a}^{x} \frac{\gamma_{\infty}(t, \lambda)}{\gamma_{\infty}(x, \lambda)}|f(t)| d t .
\end{aligned}
$$

By using the definition of the functions $\gamma_{0}(x, \lambda)$ and $\gamma_{\infty}(x, \lambda)$ (see (6)) and by applying the Cauchy- Bunyakovsky inequality we obtain

$$
\begin{aligned}
& \left|\chi_{2}(x, \lambda)\right| \leq \frac{1}{2} c_{1}(\lambda) \frac{1}{\sqrt{v(x)}}\left(\int_{a}^{x} \sqrt{\frac{v(x)}{v(t)}}\right. \\
& \left.\cdot \exp \left(-2 \int_{t}^{x} \sqrt{v(u)} d u\right) d t\right)^{1 / 2} \\
& \cdot\left(\int_{0}^{\infty}|f(t)|^{2} d t\right)^{1 / 2} .
\end{aligned}
$$

Since $t \leq x$, we get $\exp \left(-2 \int_{t}^{x} \sqrt{v(u)} d u\right) \leq 1$, and then the latter estimate for $\chi_{2}(x, \lambda)$ can be rewritten as follows:

$$
\begin{aligned}
\left|\chi_{2}(x, \lambda)\right| & \leq c_{2}(\lambda) \frac{1}{\sqrt[4]{v(x)}}\left(\int_{a}^{x} \frac{1}{\sqrt{v(t)}} d t\right)^{1 / 2} \\
& \leq c_{2}(\lambda) \frac{1}{\sqrt[4]{v(x)}}\left(\int_{a}^{\infty} \frac{1}{\sqrt{v(t)}} d t\right)^{1 / 2} .
\end{aligned}
$$

By formula (4), we get

$$
\left|\chi_{2}(x, \lambda)\right| \leq \frac{c_{3}(\lambda)}{\sqrt[4]{v(x)}}
$$

and hence if $\alpha>1$ and the coefficients of (2) satisfy the conditions (4) and (5), we have $\chi_{2}(x, \lambda) \in L_{2}(\mathbf{H},(0, \infty))$. In the case of $v(x)=x^{2 \alpha}, 0<\alpha \leq 1$, the assertion can be proved similarly.

For the function $\chi_{4}(x, \lambda)$ we will conduct the proof for the case when $v(x)=x^{2 \alpha}, 0<\alpha \leq 1$, and the coefficients of (2) satisfy condition (10). As in (37) we have

$$
\left|\chi_{4}(x, \lambda)\right| \leq c_{1}(\lambda) \gamma_{\infty}(x, \lambda) \int_{x}^{\infty} \gamma_{0}(t, \lambda)|f(t)| d t
$$

which can be rewritten as follows:

$$
\begin{aligned}
& \left|\chi_{4}(x, \lambda)\right| \\
& \quad \leq c_{1}(\lambda) \gamma_{0}(x, \lambda) \gamma_{\infty}(x, \lambda) \int_{x}^{\infty} \frac{\gamma_{0}(t, \lambda)}{\gamma_{0}(x, \lambda)}|f(t)| d t .
\end{aligned}
$$

Let us use the asymptotic behavior of the functions $\gamma_{0}(x, \lambda)$ and $\gamma_{\infty}(x, \lambda)$, for example, in the case $(\alpha+1) / 2 \alpha=n \in N$, that is, $\alpha=1 /(2 n-1)$ (see $(12))$. Setting $a(\alpha, \lambda)=((1 \cdot 3 \cdot \ldots$. $(2 n-3)) / n !) \cdot(\lambda / 2)^{n}$, we obtain

$$
\begin{aligned}
& \left|\chi_{4}(x, \lambda)\right| \leq c_{2}(\lambda) x^{-\alpha} \int_{x}^{\infty} \frac{\gamma_{0}(t, \lambda)}{\gamma_{0}(x, \lambda)}|f(t)| d t \leq c_{2}(\lambda) \\
& \cdot x^{-\alpha}\left(\int_{a}^{x}\left(\frac{\gamma_{0}(t, \lambda)}{\gamma_{0}(x, \lambda)}\right)^{2} d t\right)^{1 / 2}\left(\int_{0}^{\infty}|f(t)|^{2} d t\right)^{1 / 2}, \\
& \left|\chi_{4}(x, \lambda)\right| \leq c_{3}(\lambda) x^{-\alpha}\left(\int_{x}^{\infty}\left(\frac{t}{x}\right)^{2 a(\alpha, \lambda)-\alpha}\right. \\
& \left.\cdot \exp \frac{-2 x^{\alpha+1}\left((t / x)^{\alpha+1}-1\right)}{1+\alpha} d t\right)^{1 / 2} .
\end{aligned}
$$

Replacing variables $t=x u$, we get

$$
\begin{gathered}
\left|\chi_{4}(x, \lambda)\right| \leq c_{3}(\lambda) x^{-\alpha+1 / 2}\left(\int_{1}^{\infty} u^{2 a(\alpha, \lambda)-\alpha}\right. \\
\left.\cdot \exp \frac{-2 x^{\alpha+1}\left(u^{\alpha+1}-1\right)}{1+\alpha} d u\right)^{1 / 2}
\end{gathered}
$$

Since the inequality $\exp \left(-x^{\alpha+1}\left(u^{\alpha+1}-1\right) /(1+\alpha)\right) \leq x^{-2}$ holds for all $\alpha \in(0,1]$ and $u \in[1, \infty)$ with sufficiently large $x$, we have

$$
\begin{gathered}
\left|\chi_{4}(x, \lambda)\right| \leq c_{3}(\lambda) x^{-\alpha-1 / 2}\left(\int_{1}^{\infty} u^{2 a(\alpha, \lambda)-\alpha}\right. \\
\left.\cdot \exp \frac{-x^{\alpha+1}\left(u^{\alpha+1}-1\right)}{1+\alpha} d u\right)^{1 / 2}
\end{gathered}
$$

Hence it follows that $\left|\chi_{4}(x, \lambda)\right| \leq c_{4}(\alpha, \lambda) x^{-\alpha-1 / 2}$, and therefore $\chi_{4}(x, \lambda) \in L_{2}(\mathbf{H},(0, \infty))$. In case, where $0<\alpha \leq 1$ and $(\alpha+1) / 2 \alpha \notin N$, and where $\alpha>1$, the proof is similar.

Thus, $R_{\lambda} f \in L_{2}(\mathbf{H},(0, \infty))$ for any function $f \in$ $L_{2}(\mathbf{H},(0, \infty))$. This completes the proof.

Since the resolvent $R_{\lambda}$ is a meromorphic function of $\lambda$, the poles of which coincide with the eigenvalues of the operator $L$, the statement of Theorem 3 can be refined.

Theorem 6. If conditions (4)-(5) where $\alpha>1$ or condition (10) where $0<\alpha \leq 1$ is satisfied for (2), then the spectrum of the operator $L$ is real and discrete and coincides with the union of spectra of self-adjoint operators $L_{k}, k=\overline{1, m}$; that is,

$$
\sigma(L)=\bigcup_{k=1}^{r} \sigma\left(L_{k}\right)
$$

\section{Application}

Here we consider (2) with matrix coefficients and use the same notation as in Section 3 (note that could be considered second-order equation with block-triangular coefficients of 
a more general form [14]). Suppose that every symmetric operator $L_{k}^{\prime}$ is lower semibounded. Let $L$ be an arbitrary extension of the operator $L^{\prime}$, defined boundary condition at infinity, and $L_{k}$ an arbitrary self-adjoint extension of the operator $L_{k}^{\prime}$. If the conditions at infinity determine the Friedrichs extension $L_{k}^{0}$ of the semibounded symmetric operator $L_{k}^{\prime}$, the corresponding extension of $L^{\prime}$ will be denoted $L^{0}$. Besides, let us assume that coefficients of (2) for the problem of semiaxis are such that discrete spectrum of $L$ operator coincides with the union of discrete spectra of $L_{k}$ operators, $k=\overline{1, r}$, (sufficient conditions are specified above in Theorem 6).

Denote by $\operatorname{nul}_{a} T$ the algebraic multiplicity of 0 as an eigenvalue of $T$.

Denote by $N_{a}^{0}(\lambda)$ the number of eigenvalues $\lambda_{n}^{0}<\lambda<$ $\lambda_{e}\left(L^{0}\right)$ of the operator $L^{0}$ counted according to their algebraic multiplicities. Here $\lambda_{e}\left(L^{0}\right)$ stands for the lower bound of the essential spectrum of the operator $L^{0}$.

In [14] is set oscillation theorem of Sturm for equations with block-triangular matrix potential.

Theorem 7. Suppose the operator $L^{0}$ is generated by the differential expression $l[y]$ with matrix block-triangular potential, the boundary condition at 0 (18), and such boundary conditions at the infinity that one gets Friedrichs extensions for semibounded symmetric operators $L_{k}^{\prime}$. Then for $\lambda<\lambda_{e}\left(L^{0}\right)$ one has

$$
\sum_{x \in(0, \infty)} \operatorname{nul}_{a} Y(x, \lambda)=N_{a}^{0}(\lambda)
$$

(the sum is in those $x \in(0, \infty)$ for which $\left.\operatorname{nul}_{a} Y(x, \lambda) \neq 0\right)$.

In the same article a theorem about the connection between spectral and oscillation properties for any extension of the minimal operator is also proved. These theorems are generalizations for non-self-adjoint operators of the classical Sturm type oscillation theorems and this problem was considered for the first time.

\section{Conclusion}

In this work a resolvent is constructed for the SturmLiouville operator with a block-triangular operator potential increasing at infinite. The structure of the spectrum of such an operator is obtained.

\section{Competing Interests}

The author declared that no competing interests exist.

\section{References}

[1] M. A. Naimark, Linear Differential Operators, Part I: 1968; Part II: With Additional Material by the Author, and a Supplement by V. E. Lyantse, English Translation Edited by W. N. Everitt, Frederick Ungar, New York, NY, USA, 1969.

[2] M. A. Naimark, "Investigation of the spectrum and the expansion in eigenfunctions of a second-order non-self-adjoint differential operator on a semi-axis," Trudy Moskowskogo Matematicheskogo Obshchestva, vol. 3, pp. 181-270, 1954.
[3] V. E. Lyantse, "On non-self-adjoint second-order differential operators on the semi-axis," Doklady Akademii Nauk SSSR, vol. 154, no. 5, pp. 1030-1033, 1964.

[4] V. A. Marchenko, Sturm-Liouville Operators and Applications, vol. 22 of Operator Theory: Advances and Applications, Birkhauser, Basel, Switzerland, 1986, revised edition AMS Chelsea Publishing, Providence, RI, USA, 2011.

[5] F. S. Rofe-Beketov, "Expansion in eigenfunctions of infinite systems of differential equations in the non-self-adjoint and self-adjoint cases," Matematicheskii Sbornik, vol. 51, no. 3, pp. 293-342, 1960.

[6] J. T. Schwartz, "Some non-selfadjoint operators," Communications on Pure and Applied Mathematics, vol. 13, no. 4, pp. 609639, 1960.

[7] T. Kato, "Wave operators and similarity for some nonselfadjoint operators," Mathematische Annalen, vol. 162, pp. 258-279, 1966

[8] E. I. Bondarenko and F. S. Rofe-Beketov, "Phase equivalent matrix potential," Telecommunications and Radio Engineering, vol. 56, no. 8-9, pp. 4-29, 2000.

[9] F. S. Rofe-Beketov and E. I. Zubkova, "Inverse scattering problem on the axis for the triangular matrix potential with or without a virtual level," Azerbaijan Journal of Mathematics, vol. 1, no. 2, pp. 3-69, 2011.

[10] A. M. Kholkin and F. S. Rofe-Beketov, "On spectrum of differential operator with block-triangular matrix coefficients," Journal of Mathematical Physics, Analysis, Geometry, vol. 10, no. 1, pp. 44-63, 2014.

[11] A. M. Kholkin and F. S. Rofe-Beketov, "Green's function, resolvent, Parseval equality of differential operator with blocktriangular matrix coefficients," Operators and Matrices, vol. 8, no. 3, pp. 601-622, 2014.

[12] A. M. Kholkin, "Green's function for non-self-adjoint differential operator with block-triangular operator coefficients," Journal of Basic and Applied Research International, vol. 16, no. 2, pp. 116-121, 2016.

[13] E. C. Titchmarsh, Eigenfunction Expansions Associated with Second-order Differential Equations, vol. 2, Clarendon Press, Oxford, UK, 1958.

[14] A. M. Kholkin and F. S. Rofe-Beketov, "Sturm type oscillation theorems for equations with block-triangular matrix coefficients," Methods of Functional Analysis and Topology, vol. 18, no. 2, pp. 176-188, 2012. 


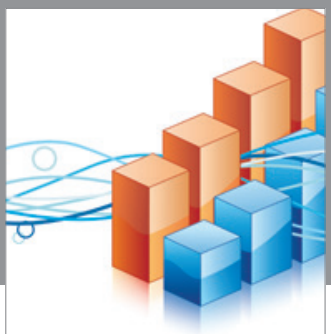

Advances in

Operations Research

vatem alat4

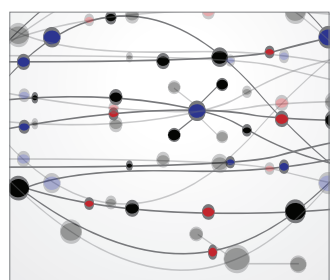

\section{The Scientific} World Journal
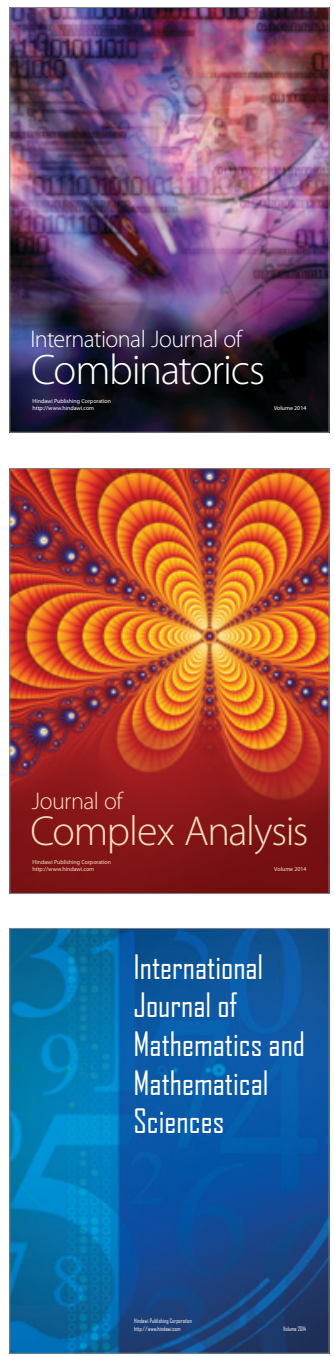
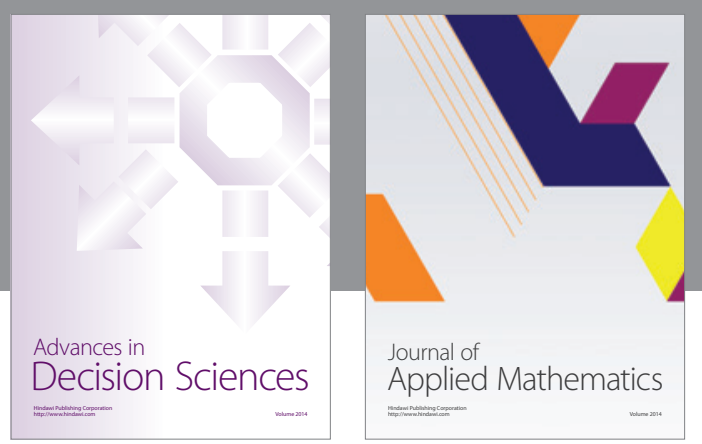

Algebra

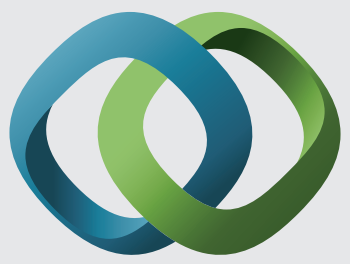

\section{Hindawi}

Submit your manuscripts at

http://www.hindawi.com
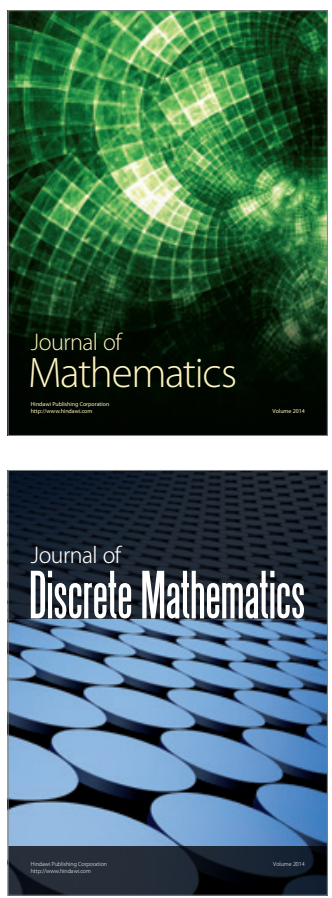

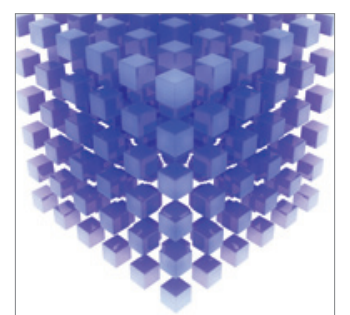

Mathematical Problems in Engineering
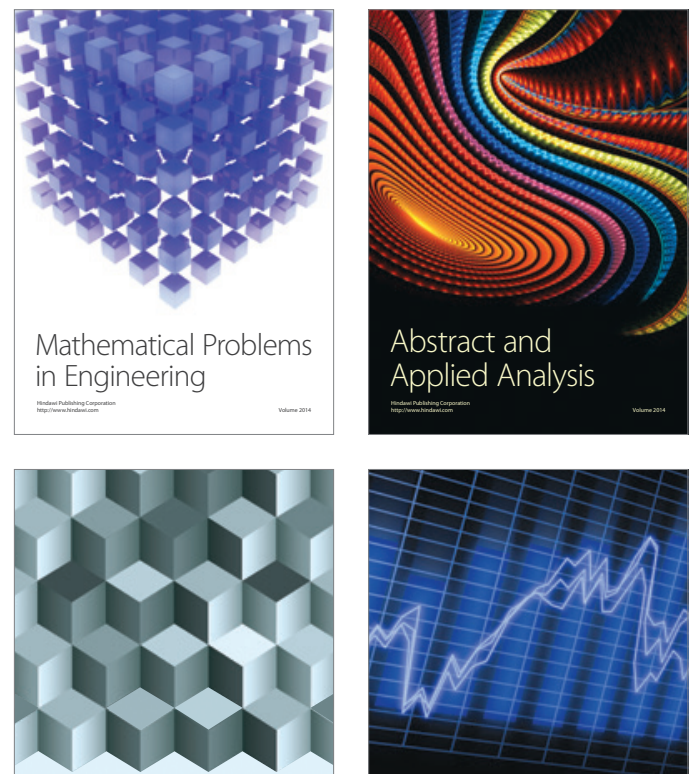

Journal of

Function Spaces

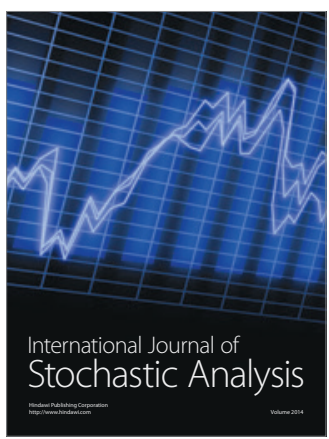

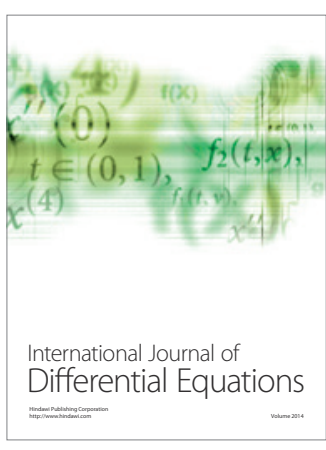
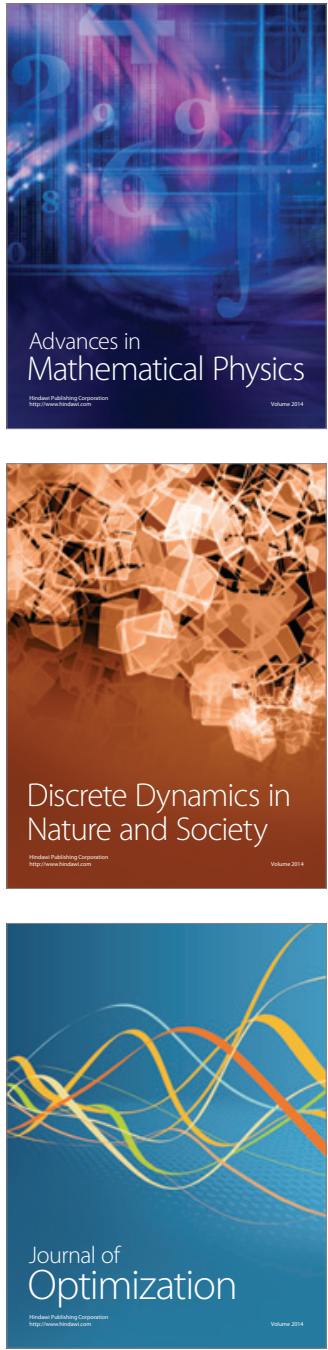